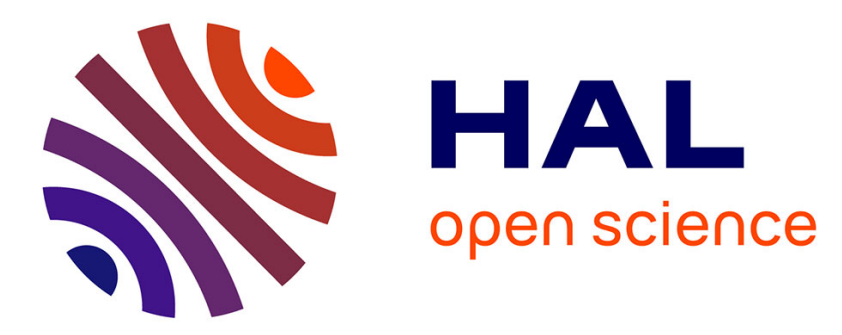

\title{
Mass entrainment-based model for separating flows
} Francesco Stella, Nicolas Mazellier, Pierric Joseph, Azeddine Kourta

\section{To cite this version:}

Francesco Stella, Nicolas Mazellier, Pierric Joseph, Azeddine Kourta. Mass entrainment-based model for separating flows. Physical Review Fluids, 2018, 3 (11), 10.1103/PhysRevFluids.3.114702 . hal02059904

\section{HAL Id: hal-02059904 https://hal.science/hal-02059904}

Submitted on 7 Mar 2019

HAL is a multi-disciplinary open access archive for the deposit and dissemination of scientific research documents, whether they are published or not. The documents may come from teaching and research institutions in France or abroad, or from public or private research centers.
L'archive ouverte pluridisciplinaire HAL, est destinée au dépôt et à la diffusion de documents scientifiques de niveau recherche, publiés ou non, émanant des établissements d'enseignement et de recherche français ou étrangers, des laboratoires publics ou privés. 


\title{
Mass entrainment-based model for separating flows
}

\author{
F. Stella, N. Mazellier, ${ }^{*}$ P. Joseph, ${ }^{\dagger}$ and A. Kourta \\ University of Orléans, INSA-CVL, PRISME, EA 4229, F45072, Orléans, France
}

(Received 23 May 2018; published 30 November 2018)

\begin{abstract}
Recent studies have shown that entrainment effectively describes the behavior of natural and forced separating flows developing behind bluff bodies, potentially paving the way to new, scalable separation control strategies. In this perspective, we propose a new interpretative framework for separated flows, based on mass entrainment. The cornerstone of the approach is an original model of the mean flow, representing it as a stationary vortex scaling with the mean recirculation length. We test our model on a set of mean separated topologies, obtained by forcing the flow over a descending ramp with a rack of synthetic jets. Our results show that both the circulation of the vortex and its characteristic size scale simply with the intensity of the backflow (the amount of mass going through the recirculation region). This suggests that the vortex model captures the essential functioning of mean mass entrainment, and that it could be used to model and/or predict the mean properties of separated flows. In addition, we use the vortex model to show that the backflow (an integral quantity) can be estimated from a single wall-pressure measurement (a pointwise quantity). This finding encourages further efforts toward industrially deployable control systems based on mass entrainment.
\end{abstract}

DOI: 10.1103/PhysRevFluids.3.114702

\section{INTRODUCTION}

Separating flows are one important source of aerodynamic losses in many industrial flows, one common example being the large shape drag of bluff bodies such as long-haul, heavy ground vehicles [1]. In this respect, understanding and controlling these flows is of primary importance for improving performances of industrial systems, in particular in the present context of increasingly stringent environmental regulations. Prototypical separating and reattaching flows developing on simple geometries, such as the backward facing step (BFS) or ramps of various shapes, have been studied extensively for several decades, providing the community with a relatively complete general understanding of their functioning. The fully turbulent separating and reattaching flow generally presents one large recirculation region [2], which extends from the separation point to the reattachment point. A separated shear layer develops between the recirculation region and the free stream, growing in thickness until it hits the wall at the reattachment point. Over the first half of the recirculation region, the separated shear layer seems to behave almost as a free shear layer [3]. Similarities include the value of its growth rate [4,5] as well as the presence of a convective instability ([6,7], among others) reminding the convection of large-scale structures reported by Brown and Roshko [8] in free shear layers. Downstream of the reattachment point, the flow slowly relaxes to a new boundary layer [9].

\footnotetext{
*nicolas.mazellier@univ-orleans.fr

${ }^{\dagger}$ Presently at Univ. Lille, CNRS, ONERA, Centrale Lille, Arts et Métiers Paris Tech, FRE 2017, Laboratoire de Mécanique des Fluides de Lille-Kampé de Fériet, Lille, F59000, France.
} 
One primary approach to mitigate aerodynamic losses caused by separating flows has focused on artificially modifying their induced pressure distribution, for example, in order to reduce drag [10]. This often comes down to controlling the shape of the recirculation region, its size, or both. Usually, the mean reattachment length $L_{R}$, defined as the streamwise distance between the mean separation point and the mean reattachment point, is considered an appropriate indicator of these geometric properties of the recirculation region. Many techniques have been proposed to modify $L_{R}$, ranging from passive devices such as vortex generators [11], to active systems such as steady suction [12] or blowing [13], pulsed jets [14], plasma actuators [15], and synthetic jets [16]. Among these methods, those based on a periodic forcing have received particular attention, because of their ability to interact with the instabilities of the separated shear layer. Many experimental [1721] and numerical [4] studies have shown that periodic actuators can be more or less effective at modifying the shape of the separation, depending on how the frequency of the forcing compares to the characteristic frequency of the natural convective instability. Actuation frequencies that are lower than this natural threshold generally create a train of coherent counterotating vortices [22], which enhances the growth of the separated shear layer and reduces $L_{R}$ [23]. Higher actuation frequencies, instead, tend to dampen shear layer instabilities, thus hindering its growth and increasing $L_{R}$.

Despite these promising results, it is an empirical fact that industrially operative, active flow control systems are rare. In particular, one critical open issue concerns their scalability. Indeed, the scaling parameters of the controlled flow are of fundamental importance, even with blackbox approaches, to guarantee reliable deployment to full-scale applications, but they are still poorly understood. Quite remarkably, this problem concerns even simple features of unperturbed separating flows. For example, some scaling laws for $L_{R}$ have been identified which seem consistent across experiments [2,24], but their practical use is often limited. Indeed, the behavior of $L_{R}$ appears to be significantly influenced by complex interactions of a large number of factors such as geometry $[25,26]$, free stream turbulence [27], the ratio between the thickness of the incoming boundary layer and the height of the step [23], and more.

In this respect, recent works suggest that simpler descriptions of the behavior of both unperturbed and controlled separating and reattaching flows might be obtained by considering the role of mass or momentum entrainment. Picking up from the theory of Chapman et al. [28], Stella et al. [5] analyzed the exchanges of mass within the separated flow behind a descending ramp, for several different values of the parameter $\operatorname{Re}_{\theta}=U_{\infty} \theta / \nu$, where $U_{\infty}$ indicates free stream velocity, $\theta$ is the momentum thickness of the incoming boundary layer, and $v$ is the kinematic viscosity of the fluid. They found that $L_{R}^{*}=L_{R} / h$, with $h$ being the height of the ramp, scales as $\operatorname{Re}_{\theta}^{m}$, where $m$ depends on the turbulent state of the incoming boundary layer. More interestingly, $L_{R}^{*}$ is approximately linearly proportional to the normalized backflow $\dot{m}_{R}^{*}=\dot{m}_{R} /\left(\rho U_{\infty} h\right), \rho$ being density, that is the flux of mass that goes through the recirculation region. In other words, Stella et al. [5] show that the characteristic length scale of separating and reattaching flows scales simply with the backflow $\dot{m}_{R}^{*}$, while the relatively complex dependency on $\operatorname{Re}_{\theta}$ can be confined to the behavior of $\dot{m}_{R}^{*}$. Results reported by Berk et al. [22] convey a similar idea. These researchers used synthetic jets to control $L_{R}^{*}$ in a BFS flow. To compare the action of the jet for four different actuation frequencies, they measured the amount of mean field momentum entrained from the free stream in a large control volume, encompassing the entire recirculation region, by the train of vortices generated by the jet. Interestingly, they found that the evolution of $L_{R}^{*}$ becomes linear when it is expressed in function of this momentum entrainment, regardless of how the actuation frequency compares to the natural convective instability of the flow. In addition, Berk et al. [22] used phased-locked PIV to analyze the convected vortices, observing that the amount of entrained momentum depends on the frequencydriven nature of the interactions between successive vortices. Once again, entrainment provides a relatively simple description of the behavior of $L_{R}^{*}$, independently of how it is itself affected by the variable controlling the flow.

Observations reported by Berk et al. [22] and Stella et al. [5] contribute to draw attention on the importance of entrainment in separating and reattaching flows. However, they do not define an entrainment-based framework for these flows. The first objective of this work is to fill this gap, by 
proposing a physics-based model of separating and reattaching flows, centered on mass entrainment. Our model approximates the separation bubble with a stationary, spanwise vortex. Its circulation $\Gamma_{V}^{*}$ and its characteristic size $L_{R}^{*}$ are both simply related to the backflow. In particular, the vortex model allows to predict the linear $L_{R}^{*}$ trend found by Berk et al. [22], in their analysis of momentum entrainment from the free stream. This appears to reduce results reported by Berk et al. [22] and by Stella et al. [5] to a single entrainment description, based on $\dot{m}_{R}^{*}$. It is pointed out that analysis of mass entrainment is preferred for its simplicity. Verifying continuity also seems a more relevant approach to the study of the behavior of $L_{R}^{*}$ (the characteristic size of a closed region) than the investigation of mean momentum transfer.

An important problem affecting any entrainment-based approach to the control of separating flows concerns the observability of entrainment. Indeed, directly measuring $\dot{m}_{R}^{*}$ (as well as any other mass or momentum flux) requires to reconstruct large portions of at least a bidimensional mean velocity field. This is unfeasible in most industrial applications, in which flow sensing can usually rely only on sparse, pointwise input, one typical example being wall-pressure information sensed by flush-mounted pressure taps. In spite of the observed linear $L_{R}^{*}$ trends, then, the reconstruction of $\dot{m}_{R}^{*}$ appears to be a real challenge for any practical application of an entrainment-based approach to flow control. Anyway, the vortex model developed in this study provides an interesting framework to tackle this problem, since the pressure distribution induced by a vortex appears to be, at least to a certain extent, related to its circulation and its topology $[29,30]$. Then the second objective of this paper is to use the vortex model to propose a correlation between $\dot{m}_{R}^{*}$ and wall pressure that might serve to develop simple, industrially deployable estimators of $\dot{m}_{R}^{*}$.

In the remainder of the paper, the symbol ${ }^{*}$ will be used to indicate normalization on $U_{\infty}$, or $h$, or both, depending on the dimensions of the normalized quantity. For developing and discussing our model, we exclusively focus on the mean flow, intended in the sense of the Reynolds averaged Navier-Stokes (RANS) equations. Accordingly, throughout this work we conform to the so-called Reynolds decomposition of the velocity field, and to its standard notation. For example, the instantaneous wall-normal velocity component $v$ will be written as

$$
v=V+v^{\prime}
$$

where $V$ and $v^{\prime}$ are the mean and fluctuating wall-normal velocities, respectively. The same decomposition and notation apply to the streamwise velocity component $u$.

The paper is organized as follows. Section II presents the vortex model of the flow, and it draws predictions for the dependencies of $L_{R}^{*}$ and $\Gamma_{V}^{*}$ with the backflow. Section III describes the experimental setup. The characteristics of the synthetic jets as well as their effects on the scaling of forced flows are discussed in Sec. IV. Section V tests the vortex model and its predictions against experimental data. The reconstruction of $\dot{m}_{R}^{*}$ from wall-pressure measurements is discussed in Sec. VI, and conclusions and perspectives are given in Sec. VII.

\section{THE VORTEX MODEL}

The insightful work of Chapman et al. [28] is a convenient starting point to discuss the role of mass entrainment in separated flows. These authors highlight that, in the mean, continuity of the recirculation region requires that the amount of mass scavenged into the reverse flow at reattachment must be balanced by the amount of mass re-entrained into the shear layer at separation. Then, although net mass entrainment through the mean separation line [31] is zero, a nonzero mass flux passes through the recirculation region, moving mass from reattachment to separation. This mass flux is usually called backflow, and many works have highlighted its huge influence on the behavior of separated flows $[4,5,23]$. It is reminded that, throughout this work, the backflow is indicated with the symbol $\dot{m}_{R}^{*}=\dot{m}_{R} /\left(\rho U_{\infty} h\right)$. The general picture sketched by Chapman et al. [28] is qualitatively confirmed by Fig. 1, which presents the streamline representation of the mean, unperturbed flow investigated in this study. This is a bidimensional, massive turbulent separation, developing behind a descending ramp geometrically similar to the one used in Stella et al. [5] 


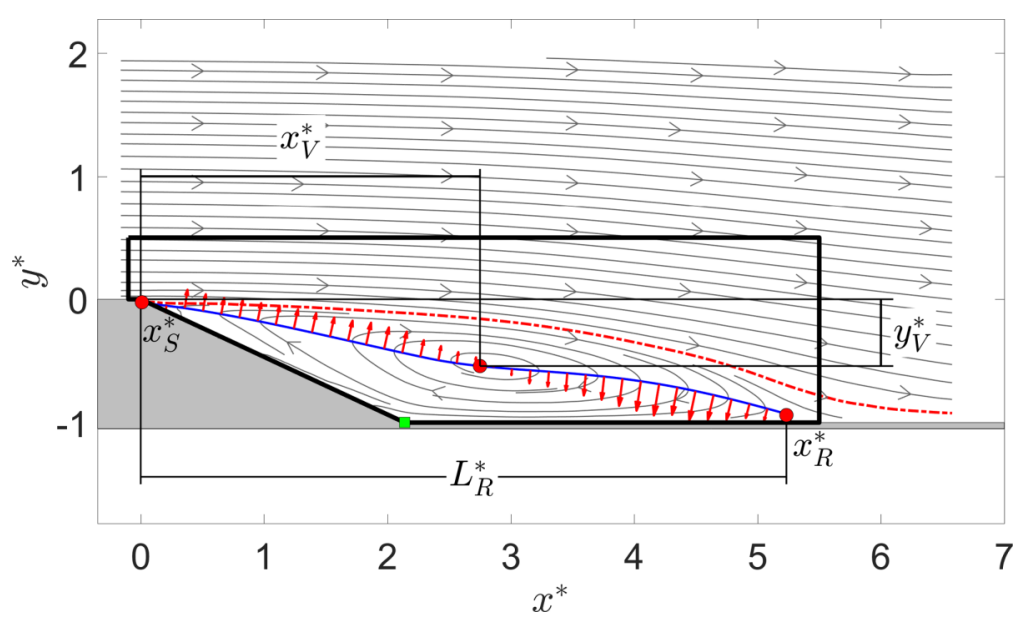

FIG. 1. Streamlines of the baseline separated flow. Symbols: (black online) closed contour $S_{V}$, used for the computation of $\Gamma^{*}$; (gray online) streamlines; (blue online) mean separation line; (red online) separating streamline; • mean separation point $\left(x_{S}^{*}, 0\right)$, center of the vortex $\left(x_{V}^{*}, y_{V}^{*}\right)$, and mean reattachment point $\left(x_{R}^{*},-1\right)$; $\square$ position of the pressure tap measuring $P_{b}$ (see Sec. VI). Red arrows represent mass fluxes $[V \cos (\phi)]$ through the mean separation line.

(see Sec. III). The mean separation point $x_{S}^{*}$ is fixed at the upper edge of the ramp and the mean reattachment point is placed at $x_{R}^{*} \approx 5.23$, which is consistent with observations reported by Kourta et al. [7]. According to Fig. 1, streamlines enter the recirculation region on $x^{*}>L_{R}^{*} / 2$, and exit it on $x^{*}<L_{R}^{*} / 2$. Reminding that by definition $U=0$ on the entire separation line, this means that the distribution of the vertical velocity $V^{*}$ along the mean separation line changes its sign at a point $\left(x_{V}, y_{V}\right)$, located at $x / L_{R} \approx 0.5$, at which $V^{*}=U^{*}=0$ (also see Stella et al. [5]). Then the backflow $\dot{m}_{R}^{*}$ can be simply computed as

$$
\dot{m}_{R}^{*}=\dot{m}_{R}^{* I N}=-\frac{\int_{0}^{s_{R}} \rho V^{-} \cos (\phi) d s}{\rho U_{\infty} h}=-\dot{m}_{R}^{* \text { OUT }}=\frac{\int_{0}^{s_{R}} \rho V^{+} \cos (\phi) d s}{\rho U_{\infty} h} .
$$

In this expression, $V^{+}$represents positive velocity values and $V^{-}$negative ones, respectively, $s$ is a curvilinear abscissa defined along the mean separation line, $s_{R}$ is the value of $s$ at reattachment, and $\phi$ is the angle between the local normal (pointing toward the free stream) and the vertical axis $Y$ (see Fig. 2). The superscripts $I N$ and OUT, respectively, indicate mass injection and extraction. It is stressed that the definition of backflow given by Eq. (2) applies to the mean field exclusively, because the net mass entrainment through the istantaneous separation line is not zero. Instantaneous mass transfer imbalances are an important feature of the reverse flow, because they are at the origin of the well-known aperiodic variation of $L_{R}^{*}$ [31]. As all unstationary behaviors of the flow, instabilities cannot be assessed with Eq. (2), nor with any of the $\dot{m}_{R}^{*}$-dependent relationships that are proposed in this work.

Our next step consists in investigating whether $\dot{m}_{R}^{*}$ is significantly related to $L_{R}^{*}$ (and possibly other quantities of the separated flow), as suggested by Stella et al. [5] and Berk et al. [22]. In this respect, it is interesting to consider Fig. 1 once again. Intuitively, streamline patterns can be interpreted as those of an elliptical, bidimensional, spanwise vortex, rotating clockwise. Since the streamlines are defined on the mean field, the vortex is also stationary and centered on $\left(x_{V}, y_{V}\right)$. Its elongated shape, fitting between the wall and the dividing streamline (see Ref. [28] among others), is approximately divided in half by the mean separation line. The upper part of the vortex corresponds to a sizable portion of the mean separated shear layer, while its lower one covers the entire mean recirculation region. This being so, $L_{R}^{*}$ is the characteristic streamwise length of the 


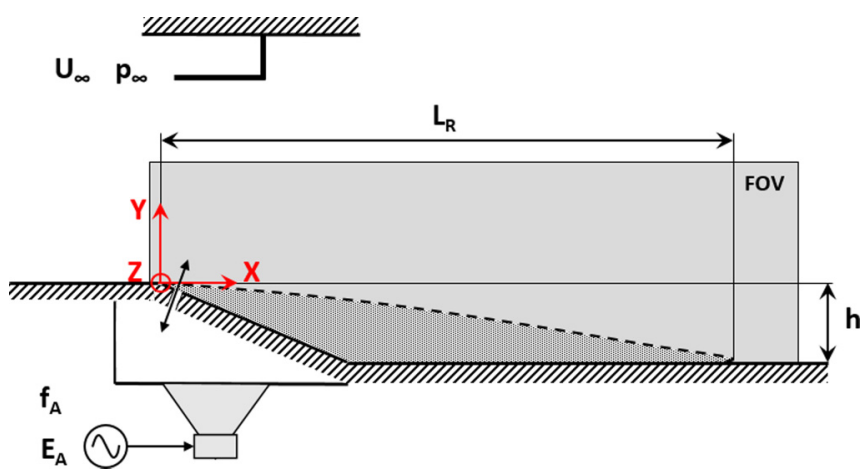

FIG. 2. Sketch of the investigated model. $X, Y$, and $Z$, respectively, indicate the streamwise, wall-normal, and spanwise axes. The shaded area represents the recirculation region, and the PIV field of view (FOV) is visualized by a gray rectangle.

vortex. These observations further suggest that $\dot{m}_{R}^{*}$ can be assimilated to the amount of mass that rotates within the vortex. If this is so, it seems reasonable to assume that $\dot{m}_{R}^{*}$ depends on the velocity distribution induced by the vortex, and hence on its characteristic size and its circulation [32]. In other words, the vortex sketched by Fig. 1 might provide a conceptual framework that relates $\dot{m}_{R}^{*}$ to the characteristic length $L_{R}^{*}$ and to the circulation of the mean flow. Then we propose to model the mean separated flow under study as the superposition of a virtual flow with no separation and an elliptical, bidimensional, spanwise vortex scaling with $L_{R}^{*}$. Let us develop this idea and its implications in more detail.

\section{A. Backflow and vortex size}

A strong relation between $\dot{m}_{R}^{*}$ and $L_{R}^{*}$ is implicit in Eq. (2), at least if it is accepted that $S_{R}^{*} \sim$ $L_{R}^{*}$, but the velocity term in that expression requires further discussion. According to our vortex approximation of the flow, an explicit prediction of the $V$ distribution along the mean separation line might be based on the odd velocity profile typical of simpler vortices (e.g., the Oseen vortex), modified as to take into account the main characteristic features of separating and reattaching flows (e.g., different turbulent intensities on the two sides of the separation line, or the no-slip condition at reattachment). Nevertheless, in the framework of the present paper, identifying the correct scaling of the $V$ distribution should be sufficient to describe the relationship between $\dot{m}_{R}^{*}$ and $L_{R}^{*}$. In particular, let us define a mean entrainment rate as

$$
v_{e, R}^{*}=\frac{\dot{m}_{R}^{*}}{S_{R}^{*} / 2} .
$$

In this expression, $v_{e, R}^{*}$ is computed on approximately half of the mean separation line, say, on $x^{*} \in\left(L_{R}^{*} / 2, L_{R}^{*}\right)$, and hence

$$
S_{R}^{*} / 2 \approx \frac{1}{h} \int_{s_{V}}^{s_{R}} d s \sim L_{R}^{*}
$$

where $s_{V}$ is the values of $s$ corresponding to $x_{V} \cdot v_{e, R}^{*}$ can be interpreted as a measure of efficiency of mass exchange through the mean separation line. In their ramp flow study, Stella et al. [5] tune the properties of the separated shear layer by progressively raising the parameter $\operatorname{Re}_{\theta}$ in the incoming boundary layer. Surprisingly, they highlight that $v_{e, R}^{*}$ remains constant, in spite of a sizable increase of shear layer growth rate caused by enhanced turbulent mixing. This finding has possibly far reaching consequences, which are only summarized here. It is well known that shear layer growth rates ultimately depend on the total entrainment rate of external fluid [33], i.e., on the efficiency of 
mass transfer from both the free stream and the reverse flow. Then if $v_{e, R}^{*}$ stays constant, it appears that turbulent mixing mostly affects efficiency of mass transfer from the free stream. If this is so, the reduction of $L_{R}^{*}$ observed by Stella et al. [5] is caused by the shear layer that, fed by more and more free-stream fluid, progressively squeezes the recirculation region to the wall. Transfer mechanisms at the separation line, however, appear to be essentially unchanged. In what follows, we make the assumption that the scenario sketched above broadly describes entrainment in any unperturbed or controlled separating flow in which the growth of the separated shear layer is driven by turbulent mixing. In particular, this allows us to consider that $v_{e, R}^{*} \approx$ const with some generality. By plugging this result in Eq. (3) and by making use of Eq. (4), one easily obtains

$$
\dot{m}_{R}^{*} \sim L_{R}^{*},
$$

which provides a simple prediction of the relationship between the backflow and the characteristic size of the vortex.

\section{B. Backflow and vortex circulation}

The second characteristic quantity of a vortex is its circulation $\Gamma_{V}$. The objective of this section is to relate circulation to the backflow, possibly with a simple expression comparable to Eq. (5). Let us begin by making the assumption that the total circulation of the flow can be modeled as

$$
\Gamma=\Gamma_{0}+\Gamma_{V},
$$

where $\Gamma_{0}$ is the circulation of the hypothetical, reattached flow. In the reference system of Fig. 2, it is both $\Gamma_{0}<0$ and $\Gamma_{V}<0$. In principle, $\Gamma_{0}$ should only depend on geometry and free stream velocity, so that $\Gamma_{0}^{*}=\Gamma_{0} /\left(U_{\infty} h\right) \approx$ const. As for what concerns $\Gamma_{V}$, it seems convenient to relate its scaling to the surface of the vortex $A_{V}$, as

$$
\Gamma_{V} \sim \omega_{z} A_{V}
$$

in which $\omega_{z}$ is a reference value of azimuthal vorticity. Mind that, since the mean flow is bidimensional, $\omega_{x}=\omega_{y}=0$. It can also be shown that in an unperturbed separating flow, the mean contribution to $\omega_{z}$ is given by the main component of mean shear:

$$
\omega_{z} \approx \frac{\partial U}{\partial y} \sim \frac{U_{\infty}}{h}
$$

This scaling law is rigorously valid only if the incoming boundary layer at separation is not too thick with respect to $h$. As an indicative threshold, we can adopt the criterion $\delta_{e} / h<0.3$ proposed by Adams and Johnston [23], in which $\delta_{e}$ is boundary layer thickness at the upper edge of the ramp. In the remainder of the paper, we will assume that Eq. (8) is also acceptable in the case of perturbed flows. Then, at least for a given geometry, Eq. (7) can be recast in nondimensional form as

$$
\Gamma_{V}^{*} \sim A_{V}^{*} \text {. }
$$

Let us now investigate the scaling of $A_{V}^{*}$. Since the stationary vortex is almost elliptical, it does not seem unreasonable to put

$$
A_{V}^{*} \approx \pi a^{*} b^{*},
$$

in which $a^{*}$ and $b^{*}$ are the dimensionless semimajor axis and the semiminor axis of the ellipse, respectively. Figure 1 suggests that the major axis can be associated with the mean separation line, so that $a^{*} \approx S_{R}^{*} / 2 \sim L_{R}^{*}$. As for what concerns $b^{*}$, its scaling can be found from simple considerations on continuity over the recirculation region. Indeed, since the backflow must remain constant across all sections of the recirculation region, the following should be verified:

$$
-b^{*} U_{R}^{*} \approx \dot{m}_{R}^{*} \approx v_{e, R}^{*} \frac{S_{R}^{*}}{2} .
$$


In this expression, $U_{R}^{*}<0$ is a characteristic streamwise velocity scale within the recirculation region. Results reported by Le et al. [9], Dandois et al. [4], and Stella et al. [5] (among others) suggest that, for a given geometry and sufficiently high Reynolds numbers, $U_{R}$ scales as $U_{\infty}$, i.e., $U_{R}^{*} \approx$ const. If $v_{e, R}^{*} \approx$ const too (see Sec. II A), dimensional analysis implies that $b^{*} \sim L_{R}^{*}$ and hence

$$
A_{V}^{*} \sim\left(L_{R}^{*}\right)^{2}
$$

By plugging this result and Eq. (5) into Eq. (9), one simply obtains

$$
\Gamma_{V}^{*} \sim\left(L_{R}^{*}\right)^{2} \sim\left(\dot{m}_{R}^{*}\right)^{2}
$$

This single expression, that links $\dot{m}_{R}^{*}$ to the main properties of the vortex $L_{R}^{*}$ and $\Gamma_{V}^{*}$, is the core of the entrainment-based framework for separating and reattaching flows that is the main objective of the present paper.

\section{EXPERIMENTAL SETUP}

The following sections will be dedicated to verify predictions provided by the vortex model, and to discuss its main assumptions. In addition, the vortex model will be used to identify a new relationship between $\dot{m}_{R}^{*}$ and the mean pressure field. Before doing so, we now introduce the experimental setup and the measuring devices used in this study.

\section{A. Setup, wind tunnel, and baseline flow}

The massive turbulent separation shown in Fig. 1 develops downstream of a salient edge, descending ramp of constant slope $\alpha=25^{\circ}$ and height $h=100 \mathrm{~mm}$. A schematic view of the ramp as well as the reference system used throughout the experiment are given in Fig. 2. Readers are referred to Kourta et al. [7] and Debien et al. [34] for further details on the setup. Measurements are taken in the S1, closed-loop wind tunnel at PRISME Laboratory, University of Orléans, France. The main test cross section is of $2 \mathrm{~m}$ by $2 \mathrm{~m}$ and $5 \mathrm{~m}$ long. The maximum reachable free stream velocity is $60 \mathrm{~ms}^{-1}$, with a turbulence intensity lower than $0.3 \%$. The ramp is placed at midheight, and it spans the entire width $w$ of the test section. This provides an aspect ratio $w / h=20$, which according to Eaton and Johnston [31] should be sufficient to guarantee that the mean separated flow is bidimensional. For the baseline, unperturbed flow, this was verified by Kourta et al. [7] with an analysis of the spanwise wall-pressure distributions at $x / h=-4, x / h=-0.5$ and $x / h=0.6$. Wall pressure was found to be homogeneous approximately over $z / w \in(-7.5,7.5)$, or on about $70 \%$ of the width of the ramp.

Throughout the experiment, the reference velocity $U_{\infty}$, measured above the upper edge of the ramp, is fixed at $20 \mathrm{~ms}^{-1}$. Following the prescriptions of Adams and Johnston [23], we use the parameter $\operatorname{Re}_{\theta}$ to assess the intensity of turbulence in the incoming boundary layer. The chosen value of $U_{\infty}$ gives $\operatorname{Re}_{\theta} \approx 2550$. To allow comparison with Stella et al. [5], $\operatorname{Re}_{\theta}$ is measured at $x / h=-9 ; y / h=0$, by integrating the mean streamwise velocity profile [35]. At $x / h=0$, it is $\delta_{e} / h \approx 0.3$, where $\delta_{e}$ is the local thickness of the incoming boundary layer.

\section{B. Measuring devices}

Since the mean flow is bidimensional, the characteristic parameters of the vortex model, which is the core of this study, can be recovered from 2D Particles Image Velocimetry (2D-2C PIV). Particle images are recorded at midspan, by two LaVision Imager LX 11M cameras (4032 px $\times 2688 \mathrm{px})$. Taking into account the overlap between the fields of view (FOV) of each camera, the total FOV covers an area of $6.3 h \times 3.2 h$ (see Fig. 2). Since the spanwise vortex is a feature of the mean field, in this work we focus on first order statistics of the flow. Then 500 independent image pairs for each tested control configuration are generally sufficient to attain statistical convergence. Laser light is provided by a $200 \mathrm{~mJ} / 15 \mathrm{~Hz} / 532 \mathrm{~nm}$ Quantel Evergreen 200-10 Nd:YAG laser, illuminating 


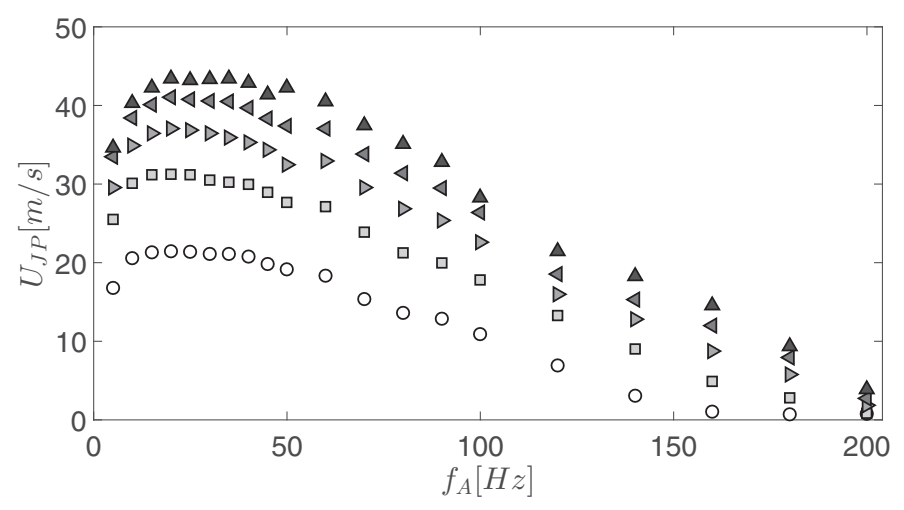

FIG. 3. Frequency response of the synthetic jet peak velocity $U_{J P}\left[\mathrm{~ms}^{-1}\right]$ at five different values of peak-topeak excitation voltage $E_{A}$. Symbols: $\circ E_{A}=0.5 \mathrm{~V} ; \square E_{A}=1 \mathrm{~V} ; \triangleright E_{A}=1.5 \mathrm{~V} ; \triangle E_{A}=2 \mathrm{~V} ; \Delta E_{A}=2.5 \mathrm{~V}$.

olive oil particles used to seed the flow. Images are correlated with the FFT, multipass algorithm of the LaVision Davis 8.3 software suit. The interrogation window is initially set to $64 \mathrm{px} \times 64 \mathrm{px}$ and then reduced to $32 \mathrm{px} \times 32 \mathrm{px}$, with a $50 \%$ overlap. These settings yield a final vector spacing of approximately $0.02 h$ in both directions.

Pointwise velocity signals are acquired by single-component, hot-wire probes. Dantec 55P15 and $55 \mathrm{P} 11$ probes are respectively used for collecting velocity profiles in the incoming boundary layer and for characterizing the response of the fluidic actuators. Signals are sampled at $f_{s}=60 \mathrm{kHz}$ for approximately $10 \mathrm{~s}$. They are subsequently low-pass filtered with a cutoff frequency $f_{c}=30 \mathrm{kHz}$.

Pressure at the lower edge of the ramp (see Fig. 1) is acquired by means of a Chell $\mu$ DAQ$32 \mathrm{C}$ pressure scanner connected to a digital acquisition unit. The scanner has a full range (FR) of $2.5 \mathrm{kPa}$ and an uncertainty of $0.25 \%$ FR. Pressure fluctuations are strongly damped by the pneumatic link between the pressure tap and the scanner [7]. Accordingly, only mean pressure variations are investigated, by averaging $3 \times 10^{4}$ samples acquired at $f_{s}=500 \mathrm{~Hz}$.

\section{FLOW FORCING}

The ability of the vortex model to represent mean separated flows needs to be tested in many different flow configurations, for example by varying ramp geometry, or parameters of the flow such as $\mathrm{Re}_{\theta}$. This task is often unpractical for the experimentalist: it requires additional measurements and time-consuming modifications of the experimental setup. In this respect, an efficient, alternative approach consists in artificially tuning the properties of the flow with an external forcing. Periodic actuators appear to be particularly well suited for this purpose, since a wide range of different flow configurations can be obtained simply by varying one or more actuation parameters (Yehoshua and Seifert [36] among others). In this study, the flow is forced with a spanwise array of three synthetic jets, designed and integrated in accordance with previous experimental and numerical studies [16,37]. Each synthetic jet is ejected through a continuous slot of width $b=1 \mathrm{~mm}$ and length $l=660 \mathrm{~mm}$, placed downstream of the upper edge of the ramp, at $x_{J}=2 \mathrm{~mm}$ (see Fig. 2). The jet is generated by a loudspeaker (Precision Devices PD.1550) and a cavity of dimensions $47 \times 25 \times 59 \mathrm{~mm}^{3}$, fitted underneath the ramp. The loudspeakers have a $380 \mathrm{~mm}$ nominal diameter and a maximum excursion of $8.2 \mathrm{~mm}$. The axis of the jet is normal to the surface of the ramp. The loudspeakers are driven by single-tone excitation signals in the form

$$
E(t)=E_{A} \sin \left(2 \pi f_{A} t\right),
$$

with $E_{A}$ being the peak-to-peak excitation voltage and $f_{A}$ the actuation frequency. Figure 3 presents the responses of the synthetic jets for $E_{A} \in[0.5,2.5] \mathrm{V}$ and $f_{A} \in[0,200] \mathrm{Hz}$. Overall, five values 


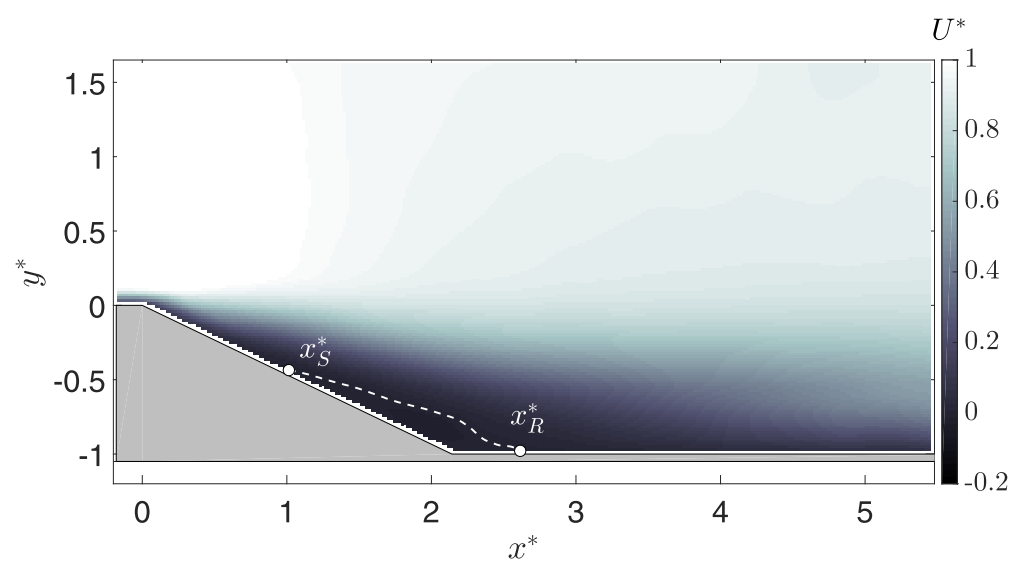

FIG. 4. Streamwise velocity field of the controlled flow at $\mathrm{St}_{A}=0.25$ and $U_{J P}^{*}=1.87$. The white dashed line (- - - - - ) indicates the mean separation line.

of $E_{A}$ and 20 values of $f_{A}$ are tested. The peak jet velocity $U_{J P}$, which depends nonlinearly on the choice of $E_{A}$ and $f_{A}$, is characterized with a hot-wire probe. Measurement settings described at Sec. III B guarantee that the frequency response of the probe is well above the largest actuation frequency tested in this study. Figure 3 shows that $U_{J P}$ is almost flat at $U_{J P, \max }$ for $f_{A} \in(20 ; 45) \mathrm{Hz}$, with slight variations with $E_{A}$, and drops significantly out of this range. $U_{J P \text {, max }}$ appears to increase monotonically with $E_{A}$, although the actuators might be approaching saturation for $E_{A} \geqslant 2 \mathrm{~V}$.

It is now important to make a clear distinction between our long-term objective of advancing flow control, and the role of the synthetic jets for our present purposes. In this study, we apply an external forcing to the flow to obtain a set of cases with different $L_{R}^{*}$, that allow to assess Eq. (13). The behavior of the actuators is, per se, not central to the present discussion. Equation (13) is an integral expression that should be independent of the working condition of the jets and of the nonlinear response of the flow. Then it seems interesting to test the robustness of Eq. (13) by spanning the working envelope of the jets along both $f_{A}$ and $U_{J P}$. According to Fig. 3, a simple way to do so is to drive the jets with variable $f_{A}$ and constant $E_{A}$. It should be noted that this is an important difference with respect to previous, comparable studies such as those reported by Chun and Sung [19] and Berk et al. [22], in which $f_{A}$ is usually changed at fixed $U_{J P}$. As a compromise between high available values of $U_{J P}$ and jet saturation, experiments are carried out with $E_{A}$ fixed at $2 \mathrm{~V}$. For convenience, in the remainder of the paper $f_{A}$ will be expressed as a Strouhal number $\mathrm{St}_{A}=f_{A} h / U_{\infty} \in(0,1)$.

Figure 4 reports an example of the mean topology of a controlled flow, which provides a general overlook of the effects of actuation. The forced shear layer grows faster than in the natural flow and is deviated toward the wall (Chun and Sung [19] among many others). This causes a sizable reduction of $L_{R}^{*}$, which is given by the displacements of both the mean reattachment point $x_{R}^{*}$ and of the mean separation point $x_{S}^{*}$. Then, unlike in the unperturbed flow, separation is no longer fixed at the upper edge of the ramp: the deviated mean shear layer interacts with the slant, causing $x_{S}^{*}$ to move downstream. It is stressed that the mean controlled flow is still approximately bidimensional. Once again, the spanwise wall-pressure distribution is found to be homogeneous on a large, central part of the width of the ramp, for all control configurations. In addition, the streamwise pressure distribution is investigated at $z / w=0, z / w=0.33$, and $z / w=-0.33$. Comparison highlights only minor discrepancies between the three samples.

Figure 5 summarizes the evolution of $L_{R}^{*}, x_{S}^{*}$ and $x_{R}^{*}$ with respect to $\mathrm{St}_{A}$. Generally speaking, observations made on Fig. 4 can be extended to the entire $\mathrm{St}_{A}$ range. Since it is $L_{R}^{*}=x_{R}^{*}-x_{S}^{*}$, a reduction of $L_{R}^{*}$ is due to both $x_{R}^{*}$ moving upstream and to $x_{S}^{*}$ being displaced downstream by the action of the synthetic jets. Uncertainties are evaluated at $-0.15 h$ for $x_{S}^{*}$ and $+0.15 h$ for $x_{R}^{*}$, 


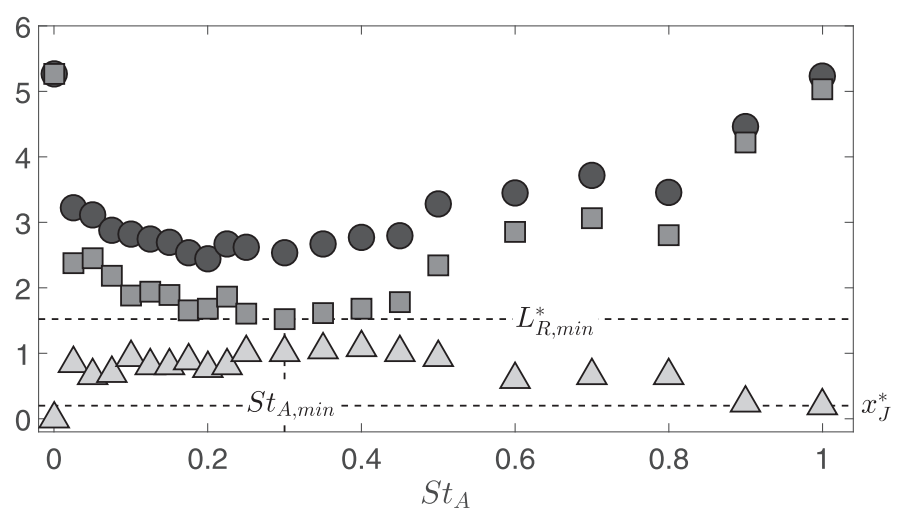

FIG. 5. Evolution with respect to $\mathrm{St}_{A}$ of $L_{R}^{*}(\square), x_{S}^{*}(\triangle)$ and $x_{R}^{*}(\bullet)$. The streamwise position of the jets is also reported, showing that $x_{S}^{*} \geqslant x_{J}^{*}$ when the synthetic jets are active.

and hence to $+0.3 h$ on $L_{R}^{*}$. This is approximately equivalent to less than $6 \%$ of the unperturbed recirculation length. Notwithstanding the $x_{S}^{*}$ displacement, the response of $L_{R}^{*}$ to actuation is in good agreement with previous observations $[17,19,22]$. Indeed, $L_{R}^{*}$ decreases strongly at low actuation frequencies, reaching its minimum $L_{R, \min }^{*} \approx 1.5$ (i.e., as much as a $75 \%$ reduction of its baseline value) for $\mathrm{St}_{A, \min } \approx 0.25$ to 0.35 . $\mathrm{St}_{A, \min }$ corresponds to $\mathrm{St}_{L}=\mathrm{St}_{A} L_{R}^{*} \approx 0.45$ to 0.55 , which matches relatively well the natural shedding frequency of the separated shear layer, estimated at $\mathrm{St}_{L} \approx 0.53$ to 0.66 by Debien et al. [6]. For $\mathrm{St}_{A}>\mathrm{St}_{A, \min }, L_{R}^{*}$ increases once again, recovering its baseline value for $\mathrm{St}_{A} \approx 1$. It is pointed out that $L_{R}^{*}$ shows a local minimum at $\mathrm{St}_{A}=0.8$. According to Chun and Sung [19], local $L_{R}^{*}$ minima can appear, typically for $\mathrm{St}_{A}>\mathrm{St}_{A \text {,min }}$, if the amplitude of the forcing is too low. In this case, large structures produced by the actuators interact with each other weakly, and are shed into the flow. This increases entrainment and produces a local reduction of $L_{R}^{*}$ on a specific $\mathrm{St}_{A}$ subrange. Figure 3 indicates that $U_{J P}$ decreases when $\mathrm{St}_{A}$ is not within $(0.1,0.25)$, so that this scenario might explain the $L_{R}^{*}$ minimum at $\mathrm{St}_{A}=0.8$.

Figure 5 shows that flow forcing drastically changes the topology of the mean flow. This allows us to assess the vortex model against a large class of different forced flows. In this respect, a first step consists in testing the relationship between $A_{V}^{*}$ and $L_{R}^{*}$ [Eq. (12)]. Direct computation of $A_{V}^{*}$ is, in general, not straightforward. Anyway, the surface of the recirculation region $A_{R}^{*}$ seems to be an adequate estimator of $A_{V}^{*}$. Indeed, on the one hand the definition of the vortex suggests that $A_{V}^{*} \approx 2 A_{R}^{*}$ (see Sec. I). On the other hand, $A_{R}^{*}$ is delimited by the mean separation line $\left(\approx 2 a^{*}\right)$ while the minor semiaxis $b^{*}$ approximately corresponds to its height. This being so, Fig. 6 reports the evolution of $A_{R}^{*}$ with respect to $\left(L_{R}^{*}\right)^{2}$, showing that available data agree well with Eq. (12). This gives a first element in support of Eq. (13) and more in general of the vortex approximation. In addition, Fig. 6 implies that $L_{R}^{*}$ remains the main characteristic length scale of the mean separating and reattaching flow. The $\mathrm{St}_{A}$-dependent characteristic length scale typical of the train of vortices generated by the synthetic jets [22] do not seem to affect the scaling of the mean flow.

\section{MASS ENTRAINMENT AND VORTEX PROPERTIES}

Findings reported in the previous section are encouraging, but the validation of the vortex model now requires to look at $\dot{m}_{R}^{*}$ more closely. Computing $\dot{m}_{R}^{*}$ is straightforward, as its definition provided by Eq. (2) can be directly applied to either halves of the mean separation line. To assess the quality of $\dot{m}_{R}^{*}$ measurements so obtained, we define the continuity error on the recirculation region as

$$
\epsilon_{R}^{*}=\dot{m}_{R}^{* I N}+\dot{m}_{R}^{* \text { OUT }}
$$




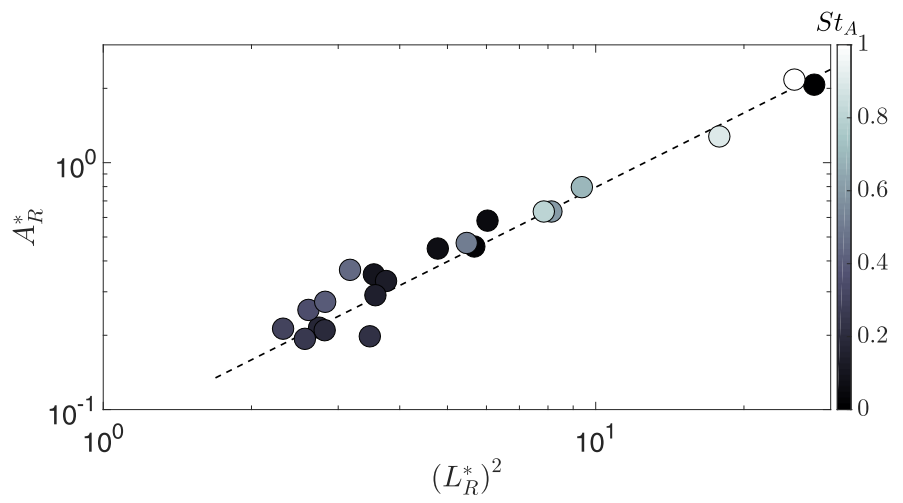

FIG. 6. Correlation between $A_{R}^{*}$ and $\left(L_{R}^{*}\right)^{2}$. The grayscale indicates the value of $\mathrm{St}_{A}$, and the dashed line (- - - - -) represents a best fit of Eq. (12) on available data. Log-log representation is used to highlight scatter for small values of $\left(L_{R}^{*}\right)^{2}$.

As shown in Fig. 7, $\epsilon_{R}^{*}$ remains below 2.5\%. Such values are close to errors reported by Stella et al. [5] and in line with the estimated uncertainties on $v_{e, R}^{*}$ and $L_{R}^{*}$ (see Sec. II and Sec. IV, respectively). Anyway, a large fraction of $\epsilon_{R}^{*}$ seems to be due to laser reflections at the wall, that produce spurious vectors in regions of the velocity field that surround the extrema of the mean separation line. Spurious vectors are more frequent in proximity of reattachment than on the slant of the ramp. In order to reduce the impact of this problem on our discussion, in what follows we only consider the estimation of the backflow obtained over the separation region, and hence we will put $\dot{m}_{R}^{*}=-\dot{m}_{R}^{* \text { OUT }}$. Figure 7 shows that $\mathrm{St}_{A}$ affects $\dot{m}_{R}^{*}$ in a similar way as $L_{R}^{*}$ (see Fig. 5). Generally speaking, $\dot{m}_{R}^{*}$ tends to decrease for $\mathrm{St}_{A}<\mathrm{St}_{A, \min }$, and to increase back to its unperturbed value for $\mathrm{St}_{A}>\mathrm{St}_{A, \min }$. Entrainment appears to be minimized at frequencies that are slightly lower than $\mathrm{St}_{A, \min }$, but this difference should be considered carefully. Indeed, even if the value of $\epsilon_{R}^{*}$ is quite homogeneous across the spanned $\mathrm{St}_{A}$ domain, its relative impact on our investigation progressively increases as $\dot{m}_{R}^{*} \rightarrow 0$, up to potentially hide the real $\dot{m}_{R}^{*}$ trend. In particular, available data indicate that $\epsilon_{R}^{*}>\dot{m}_{R}^{*}$ on $\mathrm{St}_{A} \in(0.125,0.225)$. Data points within this frequency subrange (highlighted by empty symbols in Fig. 7) will be discarded in the remainder of the paper. Figure 7 also highlights that $\dot{m}_{R}^{*}$ has two local minima, respectively at $\mathrm{St}_{A}=0.05$ and at $\mathrm{St}_{A}=0.8$. Significantly, the latter one appears to be well correlated to the $L_{R}^{*}$ local minimum shown in Fig. 5. The former one is of

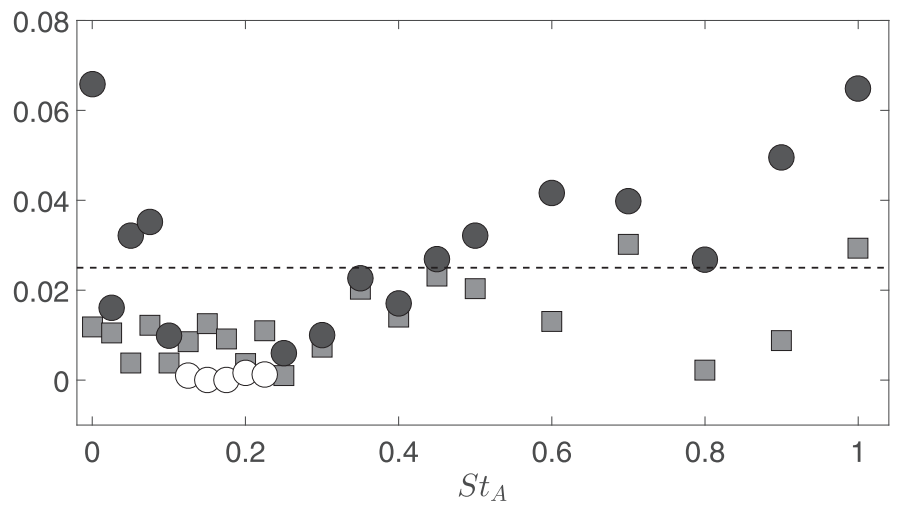

FIG. 7. Mass balance along the mean recirculation region. Symbols: $\square\left|\epsilon_{R}^{*}\right| ; \mathbf{- = - - =}\left|\epsilon_{R}^{*}\right|=0.025 ; \circ \dot{m}_{R}^{*}$. Empty symbols indicate points for which $\left|\epsilon_{R}^{*}\right|<\dot{m}_{R}^{*}$. 


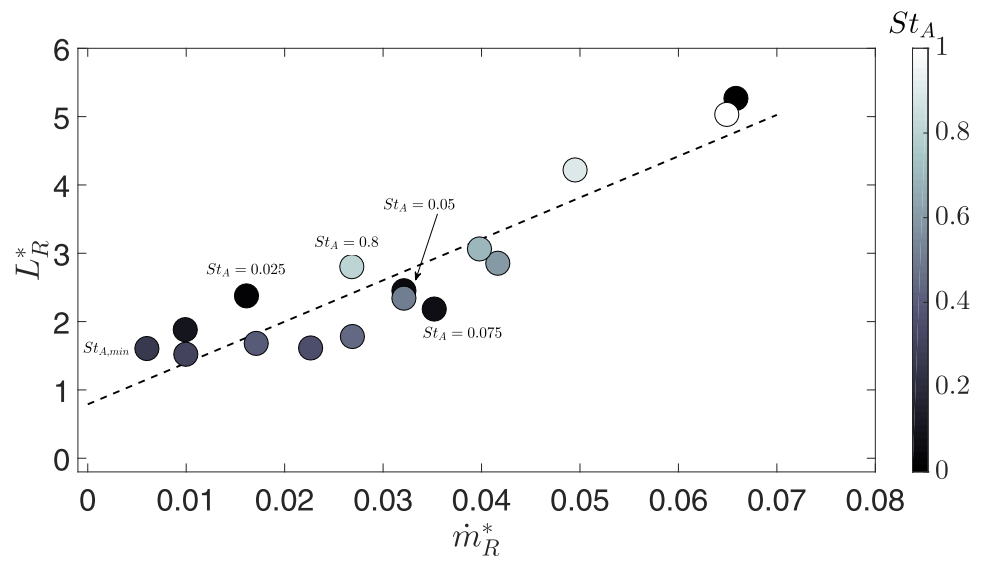

FIG. 8. Evolution of $L_{R}^{*}$ with respect to $\dot{m}_{R}^{*}$. The grayscale indicates the value of $\mathrm{St}_{A}$, and the dashed line (- - - - -) represents a linear fit as in Eq. (16). Actuation frequencies are reported for the main points discussed in Sec. V A.

more dubious interpretation: although it might be related to the trend of $x_{S}^{*}$ (see Fig. 5), it does not correspond to any clear feature of the $L_{R}^{*}$ curve. More in general, the following sections will show that points at $\mathrm{St}_{A} \in(0.05,0.075)$ verify the vortex model ambiguously. Inspection of the mean fields suggests that this behavior might be caused by increased uncertainty on $x_{S}^{*}$, on the velocity fields around reattachment, or both. Such uncertainty might be due to an interaction between the separated shear layer and the wall which is highly unsteady, compared to higher actuation frequencies. Larger statistical samples might be required to better characterize the flow in this frequency range.

\section{A. Backflow and recirculation length}

Some of the features observed in Fig. 7 suggest that $L_{R}^{*}$ might be relatively well correlated to $\dot{m}_{R}^{*}$. This is confirmed by Fig. 8, which shows that, as dimensionally predicted by Eq. (5), the $L_{R}^{*}$ trend with respect to $\dot{m}_{R}^{*}$ can be approximated by a linear model in the form

$$
L_{R}^{*}=k_{m, L} \dot{m}_{R}^{*}+L_{R, 0}^{*},
$$

where $k_{m, L}$ and $L_{R, 0}^{*}$ are estimated at approximately 60 and 0.8 , respectively, by fitting Eq. (16) on available data. The non-null value of $L_{R, 0}^{*}$, not included in Eq. (5), seems to imply that the recirculation region does not disappear for $\dot{m}_{R}^{*} \rightarrow 0$. In this paper we do not tackle this problem directly, but some of its consequences are discussed in Sec. V C. Equation (16) is in conceptual agreement with findings reported by Berk et al. [22]. Indeed, comparison of Fig. 8 with Fig. 5 proves that $\dot{m}_{R}^{*}$ is a more appropriate predictor of the evolution of controlled separating and reattaching flows than $\mathrm{St}_{A}$, providing a sensibly simpler, monotonical description of the behavior of the characteristic length scale $L_{R}^{*}$. It is stressed that the local $L_{R}^{*}$ minimum visible at $\mathrm{St}_{A}=0.8$ falls very well in the linear trend. Datapoints obtained for $\mathrm{St}_{A} \in(0.05,0.075)$ are also in reasonable visual agreement with Eq. (16). We remind that Eq. (5) does not make any hypothesis on the characteristics of the actuator. In fact, observations reported by Stella et al. [5] on the scaling of $L_{R}^{*}$ in a unperturbed flow suggest that a linear relationship between $\dot{m}_{R}^{*}$ and $L_{R}^{*}$, similar to Eq. (16), might be a general property of separating and reattaching flows assimilable to the one under study.

\section{B. Backflow and vortex circulation}

Now that predictions on the behavior of $A_{V}^{*}$ and $L_{R}^{*}$ have been verified, it remains to check whether $\Gamma_{V}^{*}$ evolves as $\left(\dot{m}_{R}^{*}\right)^{2}$, as indicated by Eq. (13). In a bidimensional flow, total circulation $\Gamma^{*}$ 


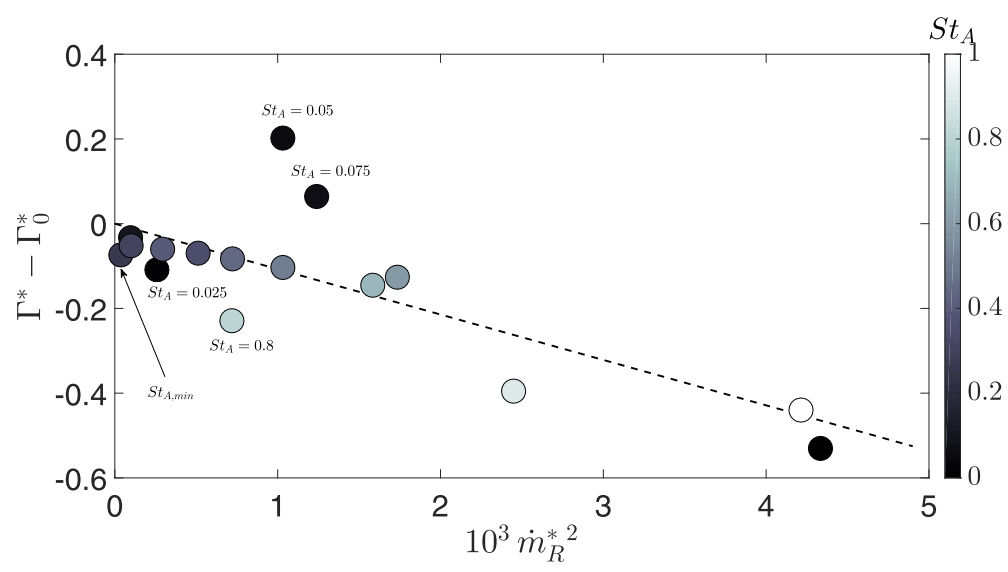

FIG. 9. Evolution of $\Gamma^{*}-\Gamma_{0}^{*}$ with respect to $\left(\dot{m}_{R}^{*}\right)^{2}$. The grayscale indicates the value $\mathrm{of} \mathrm{St}_{A}$, and the dashed line (- . - . -) represents a best fit of Eq. (13) on available data.

can be classically computed as

$$
\Gamma^{*}=\frac{1}{U_{\infty} h} \oint_{S_{V}} \boldsymbol{U} \cdot \boldsymbol{t} d s
$$

where $t$ is the local tangent to the closed contour $S_{V}$, which is presented in Fig. 1. From Eq. (6) one further obtains

$$
\Gamma_{V}^{*}=\Gamma^{*}-\Gamma_{0}^{*},
$$

in which $\Gamma_{0}^{*} \approx-5.21$ is estimated by fitting Eq. (13) onto available values of $\Gamma^{*}$ and $\dot{m}_{R}^{*}$. Figure 9 shows that computed values of $\Gamma_{V}^{*}$ verify Eq. (13) relatively well, with the exception of data at $\mathrm{St}_{A}=0.05$ and $\mathrm{St}_{A}=0.075$. In spite of these two points, it seems then safe to consider that Eq. (13) gives an acceptable description of the relationship between $\dot{m}_{R}^{*}$ and $\Gamma_{V}^{*}$.

\section{A constant entrainment rate?}

All in all, the vortex model proposed in Sec. II appears to agree well with available data. Anyway, results presented in previous sections are largely based on the hypothesis that $v_{e, R}^{*}$ can be considered constant, say, at a value $v_{e, H}^{*}$, if geometry is fixed and turbulent mixing drives shear layer growth. This assumption draws on observations made by Stella et al. [5], where changes in $L_{R}^{*}$ and $\dot{m}_{R}^{*}$ were much smaller than those reported in this study. Now, Eq. (16) shows that for very weak backflows, it is $L_{R}^{*} \rightarrow L_{R, 0}^{*} \neq 0$. Then $L_{R}^{*}$ might not vanish for $\dot{m}_{R}^{*}=0$, which would be at odds with the constant $v_{e, R}^{*}$ assumption. It seems then necessary to come back to our initial hypothesis and assess the effects of $L_{R, 0}^{*} \neq 0$. By plugging Eq. (16) into Eq. (3), simple manipulations lead to

$$
v_{e, R}^{*} \approx 2 \frac{\dot{m}_{R}^{*}}{L_{R}^{*}} \approx \frac{2}{k_{m, L}} \frac{\dot{m}_{R}^{*} /\left(L_{R, 0}^{*} / k_{m, L}\right)}{1+\dot{m}_{R}^{*} /\left(L_{R, 0}^{*} / k_{m, L}\right)},
$$

in which, for simplicity, we considered $S_{R}^{*} \approx L_{R}^{*}$. This expression suggests that $v_{e, R}^{*}$ (the efficiency of mass exchanges) is not constant as expected, but it might decrease if $\dot{m}_{R}^{*}$ becomes smaller of a certain cutoff value. Equation (19), indeed, conceptually reminds the transfer function of a high-pass filter, in the form

$$
v_{e, R}^{*} \approx v_{e, H}^{*} \frac{\dot{m}_{R}^{*} / \dot{m}_{R, \mathrm{cut}}^{*}}{1+\dot{m}_{R}^{*} / \dot{m}_{R, \mathrm{cut}}^{*}}
$$




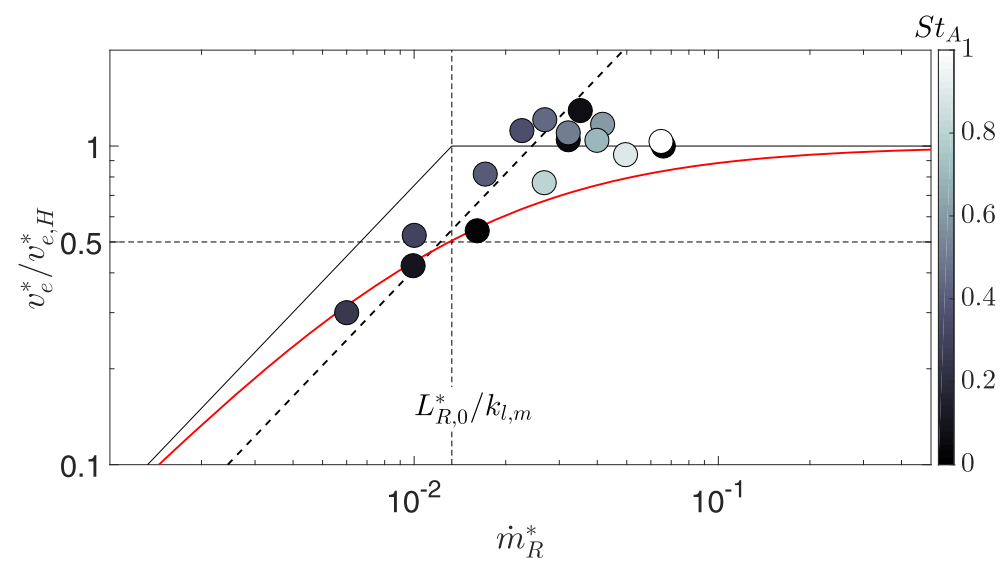

FIG. 10. Evolution of $v_{e, R}^{*}$ with $\dot{m}_{R}^{*}$. The grayscale indicates the value of $\mathrm{St}_{A}$. The characteristic of the high-pass filter defined by Eq. (19) is shown in red online (_) and its linearized form in fine, black online (— trend of $v_{e, R}^{*}$ for $\dot{m}_{R}^{*}<0.03$ is represented by a thick, dashed line (- - - - ), which was obtained by fitting a model in the form $v_{e, R}^{*} / v_{e, H}^{*}=k_{\text {filt }} \dot{m}_{R}^{*}$, where $k_{\text {filt }}$ is a constant, onto available data. The intersection of this linear fit with $v_{e, R}^{*} / v_{e, H}^{*}=0.5$ gives $\dot{m}_{R, \text { cut }}^{*} \approx 0.0123$.

where $\dot{m}_{R \text {,cut }}^{*}$ is the cutoff value, defined as the value of $\dot{m}_{R}^{*}$ for which $v_{e, R}^{*} \approx v_{e, H}^{*} / 2$. Comparing this latter expression with Eq. (19) gives $\dot{m}_{R, \text { cut }}^{*}=L_{R, 0}^{*} / k_{m, L} \approx 0.0133$ and $v_{e, H}^{*}=2 / k_{m, L} \approx 0.033$. Let us now verify if available data are consistent with this picture. Figure 10 presents values of $v_{e, R}^{*}$ in function of $\dot{m}_{R}^{*}$. $v_{e, R}^{*}$ is computed with Eq. (3) and $\dot{m}_{R}^{*}$ according to prescriptions given at the beginning of Sec. V. Generally speaking, the evolution of $v_{e, R}^{*}$ agrees relatively well with Eq. (19). For instance, available data yield $\dot{m}_{R, \text { cut }}^{*} \approx 0.0123$, which is pleasingly close to the value predicted from $k_{m, L}$ and $L_{R, 0}^{*}$. For $\dot{m}_{R}^{*}>0.03$, it is $v_{e, R}^{*} \approx v_{e, H}^{*} \approx 0.021$. This value is in good agreement with the $k_{m, L}$-based prediction given above, as well as with the entrainment rates observed by Stella et al. [5] $\left(v_{e, R}^{*} \approx 0.024 \pm 0.002\right)$. Then it appears that for large recirculation regions the efficiency of mass exchanges through the mean separation line is as insensitive to actuation as to $\operatorname{Re}_{\theta}$ and hence, broadly speaking, to the intensity of turbulent mixing. In addition, $v_{e, H}^{*}$ does not seem to be much affected by the value of the parameter $\delta_{e} / h$ either, as suggested by comparing the present experiment $\left(\delta_{e} / h \approx 0.3\right)$ with Stella et al. [5] $\left(\delta_{e} / h \approx 0.9\right)$. This being so, $v_{e, H}^{*}$ might rather depend on factors that were kept similar across experiments, in particular geometric characteristics such as the profile of the ramp, or its expansion ratio (approximately 1.1 in both cases), or both. It follows immediately that, since $v_{e, H}^{*}=2 / k_{m, L}$, the rate of variation of $L_{R}^{*}$ with $\dot{m}_{R}^{*}$ is also likely to mostly depend on geometry. For $\dot{m}_{R}^{*}<0.03$, Fig. 10 shows that $v_{e, R}^{*}$ decreases linearly, approximately of a factor 10 per every decade of $\dot{m}_{R}^{*}$ : then, the vortex model and its derived expressions [in particular, Eq. (13)] are no longer rigorously valid for small backflow values. To assess the impact of this finding on previous results, it is more practical to think in terms of $L_{R}^{*}$ : for $\dot{m}_{R}^{*}<0.03$, Eq. (16) gives $L_{R}^{*}<2.5$. Such small recirculation regions are attained for $\mathrm{St}_{A}$ approximately within $(0.05,0.5)$, which encompasses $\mathrm{St}_{A \text {,min }}$ and broadly corresponds to those actuation frequencies for which $U_{J P}^{*} \approx U_{J P, \max }^{*}$. According to Fig. 5, this means that the assumption $v_{e, R}^{*} \approx$ const is acceptable on more than half of the frequency range spanned in this study, and on about $75 \%$ of the observed variation of $L_{R}^{*}$. In addition, deviations from the vortex model should become dominant only for $\dot{m}_{R}^{*}<\dot{m}_{R \text {,cut }}^{*}$, which corresponds to $L_{R}^{*}<1.5$. Since this latter value is similar to $L_{R \text {, min }}^{*}$, the constant $v_{e, R}^{*}$ hypothesis should remain an acceptable approximation on the entire set of separated flows considered here. 


\section{AN ESTIMATOR FOR $\dot{m}_{R}^{*}$}

Findings reported in previous sections indicate that $\dot{m}_{R}^{*}$ might be a powerful variable from the point of view of flow control, since it might allow to simply reconstruct the mean topology of the flow. Unfortunately, measuring $\dot{m}_{R}^{*}$ is impossible in real-life applications, because the large velocity fields that are necessary to its computation are usually not available. In this respect, we argue that the vortex model might provide a model-based definition of simply deployable observers for $\dot{m}_{R}^{*}$, and hence $L_{R}^{*}$.

\section{A. Relating $\dot{m}_{R}^{*}$ to the pressure field}

Practical problems in sensing industrial flows make it suitable to base the estimation of $\dot{m}_{R}^{*}$ on simply accessible information. The first candidate quantity that comes to mind is wall pressure, which in most applications can be directly measured with relatively inexpensive, available on-theshelf, flush-mounted pressure taps. The mean wall-pressure distribution typical of separating and reattaching flows assimilable to the one under study is well characterized for the baseline flow (for example, see Roshko and Lau [38] and related, subsequent literature) and already documented in the case of flows controlled with synthetic jets [37]. Anyway, a quantitative link between mean wall pressure (and the pressure field more in general) and $\dot{m}_{R}^{*}$ is not self evident. It is then convenient to begin our discussion by explicitly investigating if such link exists. In this regard, the vortex model can help our reasoning, as follows. Since the vortex dominates the mean flow, it does not seem unreasonable to relate the vertical pressure force acting on the mean separation line to the circulation $\Gamma_{V}$. By invoking the Joukowski theorem, this relation can be expressed as

$$
\rho U_{\infty} \Gamma_{V} \approx \int_{S_{R}}\left(P(s)-P_{\infty}\right) d s \approx\left(P_{V}-P_{\infty}\right) S_{R} \sim\left(P_{V}-P_{\infty}\right) L_{R}
$$

in which $P(s)$ is the pressure distribution along the mean separation line, $P_{V}$ is its average value, and $P_{\infty}$ is the mean static pressure in the free stream above the descending ramp. It suits our purposes to consider that $P_{V} \approx P\left(x_{V}, y_{V}\right)$, where it is reminded that $x_{V}$ is the streamwise position of the vortex center (see Fig. 1). At least for what concerns the baseline flow, this hypothesis is supported by the odd form of the pressure gradient reported by Stella et al. [5]. By normalizing all quantities in Eq. (21), one naturally obtains

$$
\Gamma_{V}^{*} \sim C_{p, V} L_{R}^{*}
$$

In this expression, $C_{p, V}$ can be interpreted as the characteristic pressure coefficient of the center of the vortex, computed as

$$
C_{p, V}=\frac{P_{V}-P_{\infty}}{1 / 2 \rho U_{\infty}^{2}}
$$

Dependencies on $\dot{m}_{R}^{*}$ for both $\Gamma_{V}^{*}$ [Eq. (13)] and $L_{R}^{*}$ [Eq. (16)] are now well known to the reader. By plugging these expressions into Eq. (22), one simply obtains

$$
C_{p, V} \sim \frac{\Gamma_{V}^{*}}{L_{R}^{*}} \sim \dot{m}_{R}^{*}
$$

which should hold at least if $L_{R}^{*}$ is large enough with respect to $L_{R, 0}^{*}$. Equation (24) provides a first connection between the pressure field and the backflow, even if $C_{p, V}$ is generally not accessible without deeply perturbing the separated flow. We then need to go one step further in our reasoning, and relate $C_{p, V}$ to a wall-pressure value.

\section{B. The wall-normal pressure gradient within the recirculation region}

In order to introduce mean wall-pressures into Eq. (24), it is useful to look into the effects of the external forcing on the streamwise position of the center of the vortex $x_{V}^{*}$. Figure 11 shows that, 


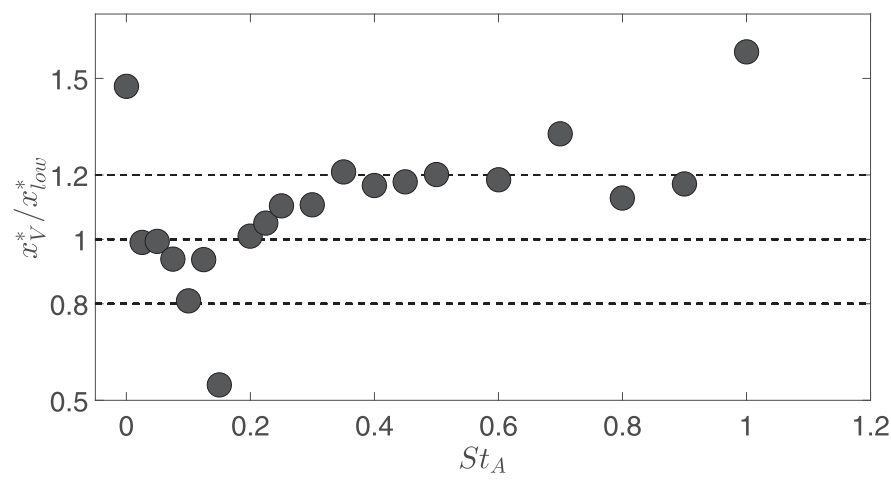

FIG. 11. Evolution of the streamwise position of the vortex $x_{V}^{*}$ with respect to $\mathrm{St}_{A}$. All available data points are included, regardless to the value of $\epsilon_{R}^{*}$ (see Sec. V).

with the exception of the baseline flow and of few controlled flows which are assimilable to it (e.g., $\left.\mathrm{St}_{A} \approx 1\right), x_{V}^{*}$ is relatively stable and similar to $x_{\text {low }}^{*}=1 / \tan (\alpha)$, which is the position of the lower edge of the ramp. This suggests that $P_{b}$, that is the wall pressure at the base of the ramp (see Fig. 1), might be related to the pressure field induced by the vortex, in particular at its center. For simplicity, let us put $x_{V}^{*} \approx x_{\text {low }}^{*}$. Then it will be

$$
C_{p, b} \approx C_{p, V}+\int_{-1-y_{V}^{*}}^{-1} \frac{\partial C_{p}}{\partial y^{*}} d y^{*}
$$

The nondimensional vertical pressure gradient $\partial C_{p} / \partial y^{*}$ can be computed with the RANS equation along the wall-normal direction, as

$$
\frac{\partial C_{p}}{\partial y^{*}} \approx-\frac{2 h}{U_{\infty}^{2}}\left(U \frac{\partial V}{\partial x}+V \frac{\partial V}{\partial y}-\frac{\partial\left\langle u^{\prime} v^{\prime}\right\rangle}{\partial x}-\frac{\partial\left\langle v^{\prime 2}\right\rangle}{\partial y}\right),
$$

where angle brackets \langle\rangle indicate ensemble averaging. It is practical to estimate the order of magnitude of $\partial C_{p} / \partial y^{*}$ with some dimensional analysis. In this respect, we remind that Eq. (11) and Eq. (12) imply that the velocity scale within the recirculation region remains $U_{\infty}$, regardless

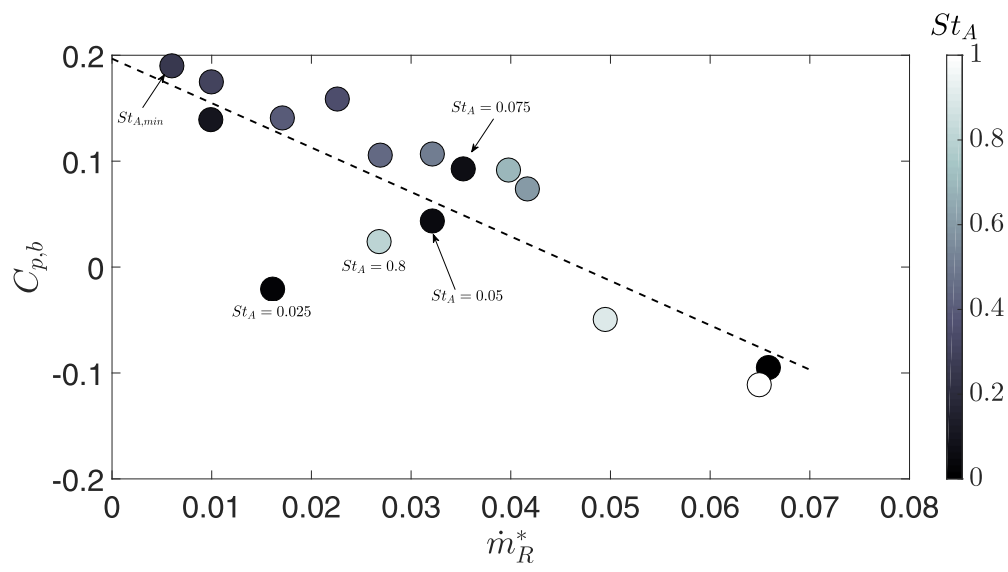

FIG. 12. Evolution of $C_{p, b}$ with respect to $\dot{m}_{R}^{*}$. The grayscale indicates the value of $\mathrm{St}_{A}$, and the dashed line (- - - - - ) represents a linear fit. 
to $U_{J P}^{*}$, at least as long as $v_{e, R}^{*} \approx$ const. If this is so, we can tentatively rely on previous works on natural separated flows to assess the order of magnitude of each term of Eq. (26). In particular, it is known that $U_{R}^{*}=O\left(10^{-1}\right)$ within the recirculation region, and that the turbulent terms will tend to cancel each other out $[4,5,9]$. Previous sections allow us to also assume that the characteristic horizontal and vertical length scales of the recirculation region will both depend on $L_{R}^{*}$, and that $V^{*} \sim v_{e, R}^{*}=O\left(10^{-2}\right)$. With these dimensional considerations in mind, Eq. (26) reduces to

$$
\frac{\partial C_{p}}{\partial y^{*}} \approx-U^{*} \frac{\partial V^{*}}{\partial x^{*}} \sim-L_{R}^{*-1} O\left(10^{-3}\right) .
$$

Since $L_{R}^{*}=O(1)$, this leads to

$$
C_{p, b} \approx C_{p, v} \sim \dot{m}_{R}^{*}
$$

The evolution of $C_{p, b}$ with respect to $\dot{m}_{R}^{*}$ is presented in Fig. 12, showing relatively good agreement with the linear trend predicted by Eq. (28). We stress the practical interest of this result: the base pressure $C_{p, b}$, obtainable with a single flush-mounted pressure tap, appears to be a reliable observer of $\dot{m}_{R}^{*}$. Since the main characteristic properties of the vortex model, including $L_{R}^{*}$, were shown to evolve approximately monotonically with $\dot{m}_{R}^{*}, C_{p, b}$ might allow to simply reconstruct many fundamental aspects of the large-scale mean topology of separating and reattaching flows, without the need for expensive and unpractical sensing systems.

\section{CONCLUSIONS}

In this study we have proposed an original model of mean separating and reattaching flows, which highlights the importance of the backflow $\dot{m}_{R}^{*}$. Our model represents the mean flow with a large spanwise vortex, covering the entire recirculation region and most of the separated shear layer. The vortex is defined by two characteristic parameters, that are its scale $L_{R}^{*}$ and its circulation $\Gamma_{V}^{*}$. In this instance, $\dot{m}_{R}^{*}$ is assimilated to the amount of mass that is entrained in rotation by the vortex. Then simple dimensional considerations allow us to predict that $L_{R}^{*}$ is linearly correlated to $\dot{m}_{R}^{*}$, and that $\Gamma_{V}^{*}$ scales as $\left(\dot{m}_{R}^{*}\right)^{2}$.

To test the relevance of the model, we have analyzed the relationship between $\dot{m}_{R}^{*}$ and the parameters of the vortex on a set of 21 different mean separated flows, obtained by forcing a single separated ramp flow with an array of synthetic jets. Each separated flow was generated with a different set of actuation parameters (frequency and amplitude). We have estimated $\dot{m}_{R}^{*}, L_{R}^{*}$, and $\Gamma_{V}^{*}$ from PIV data. All in all, these measurements agree with the relationships predicted by the vortex model, in spite of some scatter for low actuation frequencies. In particular, the linear evolution of $L_{R}^{*}$ with respect to $\dot{m}_{R}^{*}$ confirms and extends previous studies on natural and forced separating and reattaching flows, suggesting that $\dot{m}_{R}^{*}$ is a more appropriate estimator of the variation of $L_{R}^{*}$ than other flow or actuation parameters, such as the thickness of the incoming boundary layer or $S t_{A}$. This might pave the way to a universal description of separating and reattaching flows, in particular independent of the characteristics of the actuators.

As a final contribution, we have exploited the vortex model to tackle the problem of estimating $\dot{m}_{R}^{*}$ in industrial applications. By invoking the Joukovski theorem, we have proven that $\dot{m}_{R}^{*}$ is linearly correlated to the pressure at the center of the vortex, which is itself well approximated by wall pressure at the base of the ramp. As such, it appears that a single pressure measurement, simply accessible with a flush-mounted tap, might be sufficient to estimate $\dot{m}_{R}^{*}$. In turn, this might allow us to reconstruct $L_{R}^{*}$, as well as many large-scale features of the mean flow scaling with it.

In the light of these findings, our future efforts will pursue two complementary objectives. In the first place, we aim at exploiting mass entrainment to develop new model-based, possibly closed-loop separation control systems. In the second place, we would like to extend the vortex model adopted in this study, in particular by including instabilities and other unsteady phenomena. In doing so, our hope is to contribute to the development of fast, inexpensive numerical tools to predict the large-scale features of separating and reattaching flows. 


\section{ACKNOWLEDGMENTS}

This work was supported by the CNRS Groupement De Recherche (GDR) 2502 "Flow Separation Control" and by the French National Research Agency (ANR) through the Investissements d'Avenir program, under the Labex CAPRYSSES Project (ANR-11-LABX-0006-01). The authors wish to gratefully thank Prof. A. Seifert (Tel-Aviv University), for his challenging questions on the nonlinear behavior of synthetic jets, and an anonymous referee for their help in distilling the main features of the vortex model. Stéphane Loyer (PRISME, Univ. Orléans) is also acknowledged for his contribution to wind tunnel measurements.

[1] A. Seifert, T. Shtendel, and D. Dolgopyat, From lab to full scale Active Flow Control drag reduction: How to bridge the gap? J. Wind Eng. Ind. Aerodyn. 147, 262 (2015).

[2] B. F. Armaly, F. Durst, J. C. F. Pereira, and B. Schönung, Experimental and theoretical investigation of backward-facing step flow, J. Fluid Mech. 127, 473 (1983).

[3] R. L. Simpson, Turbulent boundary-layer separation, Annu. Rev. Fluid Mech. 21, 205 (1989).

[4] J. Dandois, E. Garnier, and P. Sagaut, Numerical simulation of active separation control by a synthetic jet, J. Fluid Mech. 574, 25 (2007).

[5] F. Stella, N. Mazellier, and A. Kourta, Scaling of separated shear layers: An investigation of mass entrainment, J. Fluid Mech. 826, 851 (2017).

[6] A. Debien, S. Aubrun, N. Mazellier, and A. Kourta, Salient and smooth edge ramps inducing turbulent boundary layer separation: Flow characterization for control perspective, CR Mécanique 342, 356 (2014).

[7] A. Kourta, A. Thacker, and R. Joussot, Analysis and characterization of ramp flow separation, Exp. Fluids 56, 1 (2015).

[8] G. L. Brown and A. Roshko, On density effects and large structure in turbulent mixing layers, J. Fluid Mech. 64, 775 (1974).

[9] H. Le, P. Moin, and J. Kim, Direct numerical simulation of turbulent flow over a backward-facing step, J. Fluid Mech. 330, 349 (1997).

[10] D. Barros, J. Borée, B. R. Noack, A Spohn, and T. Ruiz, Bluff body drag manipulation using pulsed jets and Coanda effect, J. Fluid Mech. 805, 422 (2016).

[11] G. Pujals, S. Depardon, and C. Cossu, Drag reduction of a 3D bluff body using coherent streamwise streaks, Exp. Fluids 49, 1085 (2010).

[12] M. Rouméas, P. Gilliéron, and A. Kourta, Drag reduction by flow separation control on a car after body, Int. J. Numer. Methods Fluids 60, 1222 (2009).

[13] J. Donovan, L. Kral, and A. Cary, Active flow control applied to an airfoil, in 36th AIAA Aerospace Sciences Meeting and Exhibit (AIAA, Reno, NV, 1997), p. 210.

[14] P. Joseph, X. Amandolése, and J.-L. Aider, Drag reduction on the $25^{\circ}$ slant angle Ahmed reference body using pulsed jets, Exp. Fluids 52, 1169 (2012).

[15] F. O. Thomas, A. Kozlov, and T. C. Corke, Plasma actuators for cylinder flow control and noise reduction, AIAA J. 46, 1921 (2008).

[16] A. Kourta and C. Leclerc, Characterization of synthetic jet actuation with application to Ahmed body wake, Sensors Actuators A 192, 13 (2013).

[17] M. Shimizu, M. Kiya, O. Mochizuki, and Y. Ido, Response of an axisymmetric separation bubble to sinusoidal forcing-Effects of forcing frequency, forcing level and Reynolds number, JSME Trans. 59, 721 (1993).

[18] L. W. Sigurdson, The structure and control of a turbulent reattaching flow, J. Fluid Mech. 298, 139 (1995).

[19] K. B. Chun and H. J. Sung, Control of turbulent separated flow over a backward-facing step by local forcing, Exp. Fluids 21, 417 (1996).

[20] A. Glezer, M. Amitay, and A. M. Honohan, Aspects of low- and high-frequency actuation for aerodynamic flow control, AIAA J. 43, 1501 (2005). 
[21] V. Parezanović, J.-C. Laurentie, C. Fourment, J. Delville, J.-P. Bonnet, A. Spohn, T. Duriez, L. Cordier, B. R. Noack, M. Abel, M. Segond, T. Shaqarin, and S. L. Brunton, Mixing layer manipulation experiment, Flow Turbul. Combust. 94, 155 (2015).

[22] T. Berk, T. Medjnoun, and B. Ganapathisubramani, Entrainment effects in periodic forcing of the flow over a backward-facing step, Phys. Rev. Fluids 2, 074605 (2017).

[23] E. W. Adams and J. P. Johnston, Effects of the separating shear layer on the reattachment flow structure. Part 1: Pressure and turbulence quantities, Exp. Fluids 6, 400 (1988).

[24] P. M. Nadge and R. N. Govardhan, High Reynolds number flow over a backward-facing step: Structure of the mean separation bubble, Exp. Fluids 55, 1 (2014).

[25] F. Durst and C. Tropea, Turbulent, backward-facing step flows in two-dimensional ducts and channels, in Proc. 3rd Intl. Symp. on Turbulent Shear Flows (Davis/CA, USA, 1981), pp. 9-11.

[26] B. Ruck and B. Makiola, Flow separation over the inclined step, in Physics of Separated FlowsNumerical, Experimental, and Theoretical Aspects, Notes on Numerical Fluid Mechanics (NNFM) (Vieweg + Teubner Verlag, Wiesbaden, 1993), Vol. 40, pp. 47-55.

[27] E. W. Adams and J. P. Johnston, Effects of the separating shear layer on the reattachment flow structure. Part 2: Reattachment length and wall shear stress, Exp. Fluids 6, 493 (1988).

[28] D. R. Chapman, D. M. Kuehn, and H. K. Larson, Investigation of Separated Flows in Supersonic and Subsonic Streams with Emphasis on the Effect of Transition, NACA Technical Report TN-1356 (NACA, Washington, DC, 1958).

[29] J. C. R. Hunt, A. A. Wray, and P. Moin, Eddies, Streams, and Convergence Zones in Turbulent Flows, Technical Report, Center for Turbulence Research Report CTR-S88 (1988).

[30] J. Jeong and F. Hussain, On the identification of a vortex, J. Fluid Mech. 285, 69 (1995).

[31] J. K. Eaton and J. P. Johnston, A review of research on subsonic turbulent flow reattachment, AIAA J. 19, 1093 (1981).

[32] G. K. Batchelor, An Introduction to Fluid Dynamics (Cambridge University Press, Cambridge, 2000).

[33] S. B. Pope, Turbulent Flows (Cambridge University Press, Cambridge, 2000).

[34] A. Debien, K. A. F. F. von Krbek, N. Mazellier, T. Duriez, L. Cordier, B. R. Noack, M. W. Abel, and A. Kourta, Closed-loop separation control over a sharp edge ramp using genetic programming, Exp. Fluids 57, 40 (2016).

[35] H. Schlichting, K. Gersten, E. Krause, H. Oertel, and K. Mayes, Boundary-Layer Theory, 6th ed. (McGraw-Hill, New York, 1968).

[36] T. Yehoshua and A. Seifert, Boundary Condition Effects on Oscillatory Momentum Generator, in 33rd AIAA Fluid Dynamics Conference and Exhibit (American Institute of Aeronautics and Astronautics, Orlando, FL, USA, 2003), p. 3710.

[37] E. Guilmineau, R. Duvigneau, and J. Labroquére, Optimization of jet parameters to control the flow on a ramp, CR Mécanique 342, 363 (2014).

[38] A. Roshko and J. C. Lau, Some Observations on Transition and Reattachment of a Free Shear Layer in Incompressible Flow, in Proceedings of the Heat Transfer and Fluid Mechanics Institute (Stanford University Press, Stanford, CA, 1965), Vol. 18, pp. 157-167. 